\title{
Seamless Handover for High-Speed Trains Using Femtocell-based Multiple Egress Network Interfaces
}

\author{
Cheng-Wei Lee, Ming-Chin Chuang, Meng Chang Chen, and Yeali S. Sun
}

\begin{abstract}
High-speed rail systems are becoming increasingly popular among long-distance travelers. With the explosive growth in the number of mobile devices, the provision of high quality telecommunication and Internet access services on high-speed trains is now a pressing problem. Network mobility (NEMO) has been proposed to enable a large number of mobile devices on a vehicle to access the Internet; however, several issues must be solved before it can be put into practice, e.g., frequent handovers, long handover latency, and poor quality of service. To resolve the above problems, we propose an LTE femtocell-based network mobility scheme that uses Multiple Egress Network interfaces to support seamless handover for high-speed rail systems, called MEN-NEMO. The results of simulations show that the proposed MEN-NEMO scheme reduces the handover latency and transmission overhead of handover signaling substantially.
\end{abstract}

Index Terms-Network mobility, high-speed train, signaling explosion, multiple network interfaces, seamless handover

\section{INTRODUCTION}

$\mathbf{H}$ IGH-speed rail systems are becoming increasingly popular among long-distance travelers because they have a number of advantages over air travel. The advantages include lower operating costs, lower fares, a more comfortable travel experience, less likelihood of weather disruptions, and lower energy consumption. With the explosive growth in the number of mobile devices, the provision of high quality telecommunication and Internet access services on high-speed trains is now a pressing problem. However, to satisfy passenger requirements in such high-speed environments, a number of challenges must be overcome, e.g., frequent handovers, simultaneous mass handovers, insufficient bandwidth, and poor quality of service [1].

The mass handover problem occurs when mobile devices perform handover from one base station (BS) to another BS simultaneously. This creates a signaling message storm and generates a large number of processing demands on the wireless link and the network components of the infrastructure. Network mobility (NEMO) [2] has been proposed to facilitate vehicle-to-infrastructure communications. It refers to the mobility of a network of communication devices (called a mobile network) that changes its attachment points to the Internet as

Manuscript received September 27, 2013, revised April 19 and September 23, 2014, accepted October 11, 2014.

Cheng-Wei Lee is with the Research Center for Information Technology Innovation, Academia Sinica, Taiwan, e-mail: jameslee@ citi.sinica.edu.tw.

Ming-Chin Chuang is with the Institute of Information Science, Academia Sinica, Taiwan, E-mail: speedboy@gmail.com.

Meng-Chang Chen is with the Institute of Information Science, Academia Sinica, Taiwan, E-mail: mcc@iis.sinica.edu.tw.

Yeali S. Sun is with the Department of Information Management, National Taiwan University, Taiwan, E-mail: sunny@im.ntu.edu.tw. one entity. All packets are transmitted over the Internet via designated mobile routers (MRs). The communication devices, called mobile network nodes (MNNs) or user equipment (UE), connect to the MR via, for example, Ethernet, WiFi or femtocell BSs. An MRs antenna is mounted outside the carriage, thereby solving the carriage penetration problem. To deal with the serious Doppler shift problem and rapid landscape changes, the MR is equipped with advanced hardware and software, such as MIMO and sophisticated signal processing techniques. In high-speed rail environment, the power consumption is not a concern because devices can be recharged. As a result, network mobility reduces the complexity of the hardware and software of MNNs. It also benefits network operators by reducing the processing and signaling overheads for authentication, authorization, accounting, and network resource management.

Choosing a wireless access technology for the train-toground link (i.e., communications between the high-speed train and the roadside eNB) is the first issue that must be addressed when implementing a network mobility scheme on high-speed rail systems. Cellular-based solutions are generating more interest than satellite Internet services because they support non-line-of-sight propagation, higher data rates and lower communication latency at less cost. Advanced standards, such as 3GPP LTE-A [24], have low handover latency and provide a data rate between $300 \mathrm{Mbps}$ and $1 \mathrm{Gps}$ on the downlink. In addition, the physical layer supports robust wireless link adaptation at speeds between 250 and $360 \mathrm{~km} / \mathrm{h}$. However, the small coverage radius of the base stations results in more frequent handovers. For instance, given a cell radius of 5 $\mathrm{km}$, mobile devices on a high-speed train traveling at 360 $\mathrm{km} / \mathrm{h}$ would experience handover every 50 seconds. Moreover, the large movement area of a high-speed train increases the likelihood of IP change, causing extra handover delay and packet loss.

A major disadvantage of using a single egress network interface is that, the user equipment (UE) suffers from a transient transmission disruption during handover; and this problem becomes more serious as the handover frequency increases. As an alternative, multiple interfaces (including multiple MRs) were used initially to fully utilize the wireless link diversity (a.k.a., antenna diversity) and thereby improve the link quality. Recently, multiple interfaces have been proposed to coordinate and facilitate seamless handover. In this paper, we focus on seamless handover for high-speed trains and propose a network mobility protocol with multiple egress network interfaces, called MEN-NEMO.

The remainder of this paper is organized as follows. Section II provides a review of related works on network mobility 
design and multiple egress network interfaces. In Section III, we describe the operations of the proposed mechanism; and in Section IV, we analyze its performance. Section V contains some concluding remarks.

\section{RELATED WORK}

\section{A. Network Mobility Design}

A number of solutions have been proposed for network mobility on different protocol layers. In general, higher level mobility management requires less infrastructural support, but it entails end-system support, additional signaling and an encapsulation overhead. In contrast, lower level mobility management provides more efficient signaling and handover operations; and it requires less involvement of end-systems by introducing heavy infrastructure dependency [5].

SIP-NEMO [6] and SIP-NMG [7] are designed for Session Initial Protocol (SIP)-based applications. The MR on the vehicle translates SIP signaling for the MNN. HarMoNy [5] integrates the Host Identity Protocol (HIP) with Mobile IPv6 by exploiting HIP-enabled MNNs. The above protocols are designed for specific applications or they require new functionality on the MNNs, which is a disadvantage in widely deployed applications. The network mobility basic support protocol [8], which establishes a bi-directional tunnel between the MR and its home agent, provides mobility transparency for the MNNs and allows them to use native applications without modification. However, in the context of high-speed rail systems, more advanced wireless access technology should be adopted to provide better signal quality; and more efficient handover mechanisms are required to ensure un-interrupted communications.

\section{B. Multiple Egress Network Interfaces}

Multiple egress network interfaces improve the link quality and handover performance of mobile devices in vehicles. For example, the Mobile Access Router (MAR) infrastructure [9] integrates multiple wireless access technologies and exploits network diversity to provide a high quality communication channel for MNNs. However, a single egress network interface for each wireless access technology is still not sufficiently robust or reliable [3]. In this paper, we use equivalent egress network interfaces to share the communication load and perform handovers cooperatively.

Our previous work [3] takes advantage of a trains length to deploy multiple MRs in WiMAX networks. A network mobility management protocol is designed on the IPv6 network. An egress network interface in the head carriage and another one in the tail carriage perform handover alternately and transmit the data traffic of the mobile network. The scheme provides seamless handover for the MNNs on a high-speed train. Later, Tian et al. [4] also utilize the same concept as [3] and implement the MR by using LTE relay stations. This architecture is called Train Relay Station (TRS) and it requires the transfer of all the information (e.g., the identities) about the active UEs on the train (i.e., the MNNs) when performing handover. The TRS handover protocol employs bi-casting from the serving gateway $(\mathrm{S}-\mathrm{GW})$ to the serving evolved Node B
(eNB) and the target eNB during handover. The objective is to eliminate the data forwarding delay between the serving eNB and the target eNB. A femtocell-based network mobility solution and handover scheme is proposed in [10]. The femtocell provides IP-based encapsulation communications for the MNNs so that only the femtocell BSs information has to be transferred and processed when performing handover. However, the authors of [10] do not provide details about the signaling and configuration. Moreover, the femtocell on each carriage works independently without utilizing the link diversity to improve the signal quality, and it also does not consider the effect of the overlapping area.

\section{Information Raining}

Huang et al. [21] proposed an antenna assignment algorithm for maximum ratio combining. Moreover, this algorithm can also be applied to the information raining system. Sue et al. [22] proposed the network coding scheme for improving the performance of the high-speed train in IEEE 802.16 relay networks. Ho and Valaee [23] designed a novel system architecture that enables high-speed Internet access in railway systems. In addition, they used packet fragment and repeater relay schemes to enhance the system throughput. The goals of these studies [21-23] focus on high throughput in a highspeed train environment without taking handover problem into account, while this paper focuses on the handover procedure to allow low handover latency, low packet loss and signaling overhead.

\section{THE PROPOSED SCHEME}

Next, we present the system architecture of the network mobility (NEMO) protocol for high-speed trains. It is based on LTE femtocell technology with multiple egress network interfaces. We solved the routing optimization and IP mobility issues of NEMO and nested NEMO in our previous works [3] [18] [19]. We used the pre-handover mechanism to reduce the handover latency and provided a buffer management mechanism to resolve the packet loss problem in NEMO. Moreover, we adopted the ad hoc routing scheme to achieve the routing optimization of transmission path in nested NEMO. In this paper, we focus on the seamless handover procedure between the serving eNB and the target eNB.

\section{A. The System/Core Network Architecture}

In Fig. 1, an enhanced femtocell base station (called an enhanced HeNB) is deployed on a train to serve the user equipment (UE) on the train (i.e., the MNNs) as an eNB. The Mobility Management Entity (MME) and the Serving Gateway $(\mathrm{S}-\mathrm{GW})$ handle the UEs mobility events. The $\mathrm{MME}_{U}$ and $\mathrm{S}-\mathrm{GW}_{U}$ are responsible for the UEs mobility events and packet forwarding, respectively; the $\mathrm{MME}_{f}$ and $\mathrm{S}-\mathrm{GW}_{f}$ are responsible for the enhanced HeNBs mobility events and packet forwarding, respectively. Under the LTE standard, a HeNB connects to the operators core network via a public network over a secure IP-based connection (e.g., IPsec). The proposed scheme configures the egress interface(s) of the 
enhanced HeNB to communicate with the $\mathrm{MME}_{U}$ and $\mathrm{S}-\mathrm{GW}_{U}$ via the IP connection in an LTE wireless access network. In other words, the enhanced HeNBs egress interface acts as a UE that connects to the serving eNB on the ground and is served by the $\mathrm{MME}_{f}$ and the $\mathrm{S}-\mathrm{GW}_{f} 1$. As a result, when a request (e.g., a call) is destined for the UE, it is forwarded from the $\mathrm{MME}_{U} / \mathrm{S}-\mathrm{GW}_{U}$ to the enhanced HeNB via the S-GW 1 and the serving eNB. Note that, for clarity, some interfaces (e.g., between $\mathrm{MME}_{f}$ and the eNB under $\mathrm{S}-\mathrm{GW}_{f} 2$ ) are not shown in Fig. 1.

The detailed protocol stacks of the S1-MME control plane and S1-U user plane between the enhanced HeNB and the $\mathrm{MME}_{U}$ and $\mathrm{S}-\mathrm{GW}_{U}$ [11] are shown in Figs. 2 and 3 respectively, including the underlying S1-U user plane. The control signal and data packets for the UE are IP-encapsulated (and decapsulated) by the enhanced HeNB. Consequently, the $\mathrm{MME}_{f}$ and the $\mathrm{S}-\mathrm{GW}_{f} 1$ only have to exchange and process the context of the enhanced HeNB, including the network resource reservation and admission control. In addition, when the enhanced HeNB performs handover from the serving eNB to the target eNB [11], or when the anchor S-GW of the enhanced HeNB is changed from the $\mathrm{S}-\mathrm{GW}_{f} 1$ to the $\mathrm{S}-\mathrm{GW}_{f} 2$ (i.e., Tracking Area Update) [12], the signal only includes the information about the enhanced HeNB. Note that if the IP address of the enhanced HeNBs egress network interface is changed after handover, the enhanced HeNB should reregister with the $\mathrm{MME}_{U}$ and the $\mathrm{S}-\mathrm{GW}_{U}$, as stipulated in [13]. The network configuration proposed in this paper does not require modification of the $3 \mathrm{GPP}$ standard. The performance can be further improved in some circumstances. For example, if the enhanced HeNB is operated by the same operator as the eNBs, the connection between the enhanced HeNB and the $\mathrm{MME}_{U} / \mathrm{S}-\mathrm{GW}_{U}$ is naturally secured by the lower layer bearers. As the connection does not require extra encryption, the processing, signaling and packet header overheads are reduced. Moreover, if the S-GW $\mathrm{GW}_{f}$ supports direct forwarding to the $\mathrm{MME}_{U}$ and $\mathrm{S}-\mathrm{GW}_{U}$ without going through the $\mathrm{P}-\mathrm{GW}_{f}$, the transmission delay can be reduced further.

Note that our architecture is compatible with the 3GPP standard. Therefore, the security functionality of the 3GPP

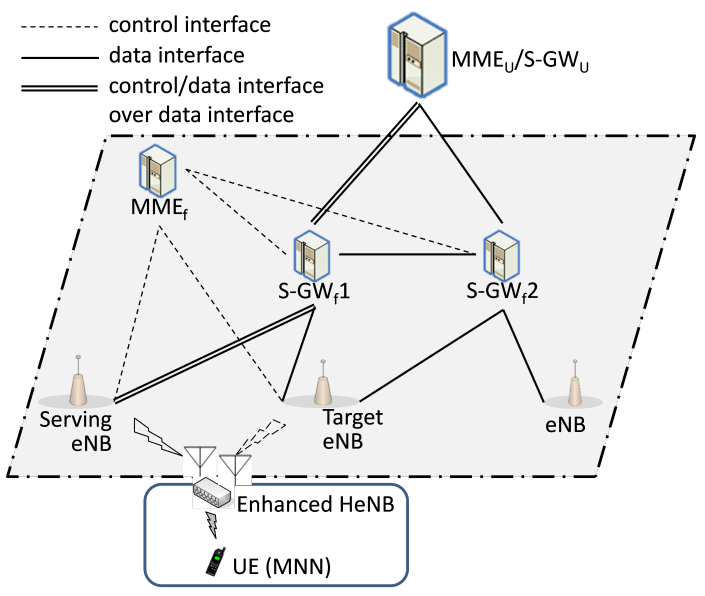

Fig. 1. The proposed system architecture

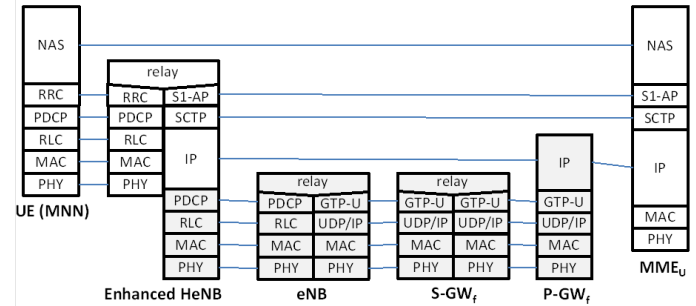

Fig. 2. The S1-MME control plane over the S1-U user plane

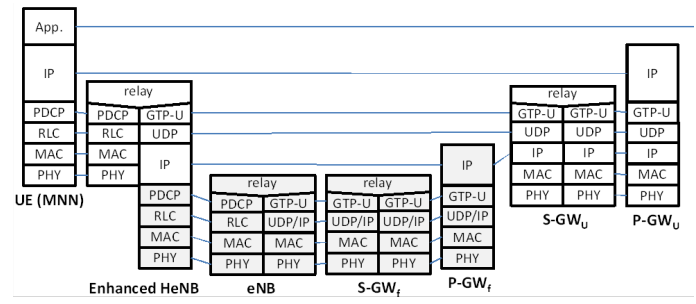

Fig. 3. The S1-U user plane over the S1-U user plane

standard can be applied directly in our architecture. In the architecture, the high-speed train acts as a big mobile device with multiple distributed antennas. The MNN performs the security operations (e.g., authentication, registration, etc.) when it connects to the Internet through the enhanced HeNB in the first time, which will keep the security information of the MNN. Afterward, the enhanced HeNB performs the security operations on behalf of MNNs until the MNN leaves the highspeed train.

\section{B. The Network Architecture on a Train}

Figure 4 illustrates the proposed network architecture for a high-speed train. The architecture, which is a modified version of that presented in [3], uses an enhanced HeNB to implement the MR. The UE on the train attaches to the enhanced HeNB via the access point in the carriage. Wired and wireless access technologies like Ethernet, WiFi, 2G or $3 \mathrm{G}$ can also be adopted to provide data communication and telecommunication services to the MNNs. The enhanced HeNBs egress interface has two antennas, one deployed on the head carriage and one on the tail carriage to ensure good signal quality and support seamless handover. The egress interface is allocated one IP address, which is used by the enhanced HeNB to connect to the $\mathrm{MME}_{U}$ and the S-GW${ }_{U}$. The $\mathrm{MME}_{f}$, S$\mathrm{GW}_{f}$ and $\mathrm{P}-\mathrm{GW}_{f}$ recognize the enhanced HeNB by its ID (e.g., the IMSI or the TMSI) and tunnel its data to the serving eNB (according to the eNB ID). The approaches in [3] and [4] utilize the same framework for seamless handover on highspeed trains. The enhanced HeNB continuously monitors the communication conditions of the head antenna for handover decisions.

When a pre-defined condition is reached, such as the signal strength threshold, the enhanced HeNB uses the head antenna to perform handover to the target eNB; meanwhile, it transmits uplink and downlink traffic via the tail antenna, which is still attached to the serving eNB. After the enhanced 


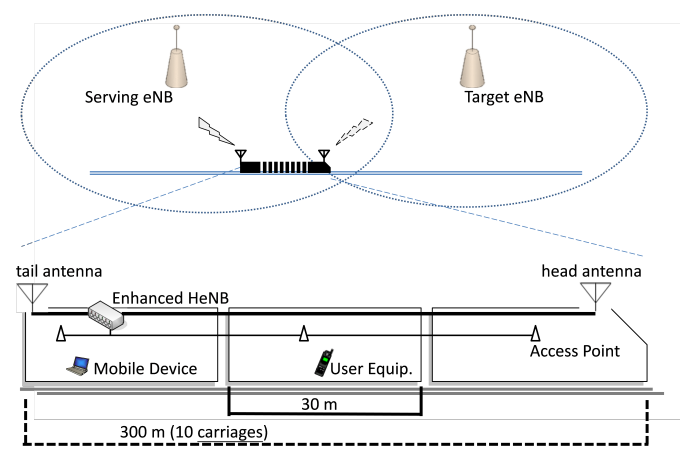

Fig. 4. The multiple egress network interfaces on a train with ten carriages

HeNB completes the handover, the uplink and downlink traffic transmissions are seamlessly transferred to the head antenna via the target eNB (i.e., the new serving eNB). On a 300 meter high-speed train traveling at $360 \mathrm{~km} / \mathrm{h}$, it takes about 3 seconds for the tail antenna to reach the same handover condition as the head antenna. That is sufficient time for the enhanced HeNB to complete handover by using the head antenna.

As a result, the UEs communications are not interrupted during handover. Even if handover using the head antenna fails, the enhanced HeNB will use the tail antenna to perform handover again. The head antenna or the tail antenna performs the handover procedure successfully, and then the communications of MNNs will not be interrupted. Next, we describe the seamless handover procedure in detail.

\section{Seamless Handover Procedure}

The design of the seamless intra-MME/S-GW handover procedure of MEN-NEMO is based on the 3GPP specification [11]. In addition, the movement trajectory of the train is fixed in high-speed rail environment. Therefore, target eNBs can be known in advance even if the signal strength measurements are very noisy. Figure 5 shows the detailed handover signaling diagram. The steps are described as follows.

1) The enhanced HeNB periodically sends a Measurement Report to the serving eNB. The report contains signaling information, such as the received signal strength indication (RSSI) of the head antenna for handover decisions.

2) The serving eNB prepares to perform the handover procedure when it receives the RSSI report of the target eNB from the head antenna. Based on the information in the Measurment Report and Radio Resource Management (RRM), the serving eNB makes decisions about handoff to the enhanced HeNB. We discuss the detailed handover decision policy in the next subsection.

3) The serving eNB issues a Handover Request with the necessary information to prepare for handover to the target eNB, and Admission Control is used to increase the likelihood of a successful handover. The target eNB replies to the serving eNB with a Handover Request Acknowledgement message to confirm the handover.

4) The serving eNB sends an Radio Resource Control (RRC) Connection Reconfiguration to the enhanced HeNB. The message contains the channel access parameters that can be used to attach to the target eNB.

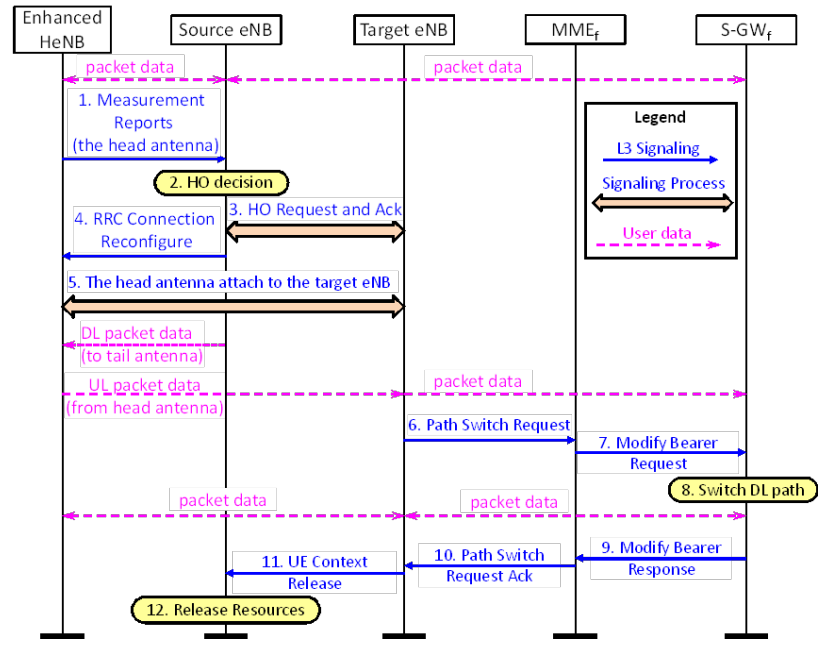

Fig. 5. The proposed handover procedure

5) When the enhanced HeNB receives the RRC Connection Reconfiguration, it synchronizes with the target eNB and uses the head antenna to access the target cell. After the head antenna is attached to the target eNB, the enhanced HeNB forwards uplink traffic to the target eNB via the head antenna. Meanwhile, the serving eNB continues sending packets to the enhanced HeNB via the tail antenna, and the SN Status Transfer is cancelled.

6) The target eNB sends a Path Switch Request to the $\mathrm{MME}_{f}$ to inform it that the enhanced HeNB has changed to a different cell.

7) The $\mathrm{MME}_{f}$ sends a Modify Bearer Request with the target eNBs ID to the $\mathrm{S}-\mathrm{GW}_{f}$.

8) The $\mathrm{S}-\mathrm{GW}_{f}$ switches the downlink data path to the target side. As a result, the downlink packets are now forwarded to the enhanced HeNB via the target eNB and the head antenna.

From steps 9) to 11), the Modify Bearer Response, Path Switch Request Acknowledgement and UE Context Release messages are identical to the standard. Finally, in step 12) the serving eNB releases the unused resource.

In the MEN-NEMO scheme, the S-GW ${ }_{f}$ switches the forwarding tunnel end-point for downlink traffic from the serving eNB to the target eNB once the head antenna has attached to the target eNB. This step does not involve extra bandwidth usage. The signaling delay between the target eNB and the $\mathrm{S}-\mathrm{GW}_{f}$ and the one-way delay in the opposite direction are negligible and do not affect the handover performance. This is because the signaling delay is completely overlaid by the packet transmission time of head and tail antennas.

In the standard, each UE only uses a single wireless interface to connect to eNB and individually and independently performs the handover procedure. This handover procedure of the standard will cause longer handover latency and higher handover signaling overhead. The main difference between standard and our scheme is from steps 5 to 8 . In MEN-NEMO, the communications is not disrupted during handover since there are multiple distributed antennas on the train. The tail antenna still receives the packets from the serving eNB when the head antenna performs the handover procedure with the 
target eNB. One difference between the MEN-NEMO scheme and TRS [4] is that the latter employs bi-casting, which requires additional network resources to handle redundant traffic. The amount of traffic may be large for all the UEs on the train.

\section{Discussion of Handover Failure}

We discuss how the tail antenna will handle the handover failure of the head antenna situation. Basically, the head antenna repeatedly performs the handover procedure until the handover procedure of the head antenna is successful. At this moment in time, the tail antenna still connects to the serving eNB. In the worst case, the tail antenna takes over the handover procedure of the head antenna, when the tail antenna will hand out the coverage of the serving eNB and the head antenna still cannot connect to the target eNB.

The real handover failure is happened when the head and tail antennas both execute the handover procedure unsuccessfully. When the noise is low, Tian et al. [4] showed in the section 4 that it can be ignored. It says that 1) inter-carrier interference (ICI) and noise component could be averaged out and have no effect on the final average signal strength in small-scale fading. 2) Moreover, the probability of the handover failure is very low (i.e., $10^{-3}$ to $10^{-5}$ ) when the train equips two antennas at the speed of $360 \mathrm{~km} / \mathrm{h}$. When the noise is loud and causes transmission failure, retransmission can be done in the proposed scheme.

\section{E. Handover decision policy}

In existing works, most handover decision policies are based on the RSSI variant. The UE (or enhanced HeNB) scans the eNB of the neighbor cell list and usually selects the eNB that has the best RSSI connection. However, the UE (or enhanced HeNB) could execute unnecessary handover operations (i.e., extra auto-configuration time for the IP address). This would increase the signaling overhead and the probability of service interruption when the UE (or enhanced HeNB) hands over to a different IP domain.

To prevent unnecessary frequently time-consuming IP reconfiguration and reduce potential packet delay and loss, we add more constraints to the handover decision policy. In Step 1 of Fig. 5, the enhanced HeNB sends a Measurement Report, which includes the candidate target $\mathrm{eNB}(\mathrm{s})$, to the serving eNB. At that point, the serving eNB checks the RSSI(s) of the candidate target $\mathrm{eNB}(\mathrm{s})$ and determines if the target $\mathrm{eNB}(\mathrm{s})$ and the serving eNB are in the same IP domain. If a candidate target eNB has a high RSSI and the same IP domain as the serving eNB, it is chosen first. This is because the enhanced HeNB only has to wait for the path switch time (i.e., Steps 6 to 10 in Fig. 5) without an IP change when it performs handover under the same IP domain.

\section{PERFORMANCE ANALYSIS}

In this section, we evaluate the handover latency, user satisfaction, the total size of handover signaling messages, and packet loss in different schemes. We simulate the performance of the MEN-NEMO scheme using network simulator
3 (NS3) [17] and compare it with the performance of the LTE-individual and TRS schemes. Each simulation result is the average of thirty runs. The value of error bar is obtained from a $90 \%$ confidence interval.We consider two simulation scenarios, as shown in Figure 6. The first (i.e., Fig. 6(a)) is a simple environment in which the enhanced HeNB is served under the same IP domain and does not need to change its IP address when a handover occurs. By contrast, Fig. 6(b) shows a complex environment in which the target eNB(s) may belong to a different IP domain. Therefore, the enhanced HeNB needs to change its IP address when it hands over to a different IP domain. In the simulation setting, we assume that the network environment allows line-of-sight communications. Table I shows the key parameters used in the system level simulation [20]. In the figures, Sim means the results were obtained from the simulations, and Ana means the results were obtained via numerical analysis.

\section{A. Performance Metrics}

The performance metrics are summarized as follows.

(1) Handover latency: The disruption time is computed from the time when the train begins the handover procedure from its serving eNB to the target eNB.

(2) User satisfaction: The satisfaction score function utilizes reward and punishment to evaluate the satisfaction levels of UEs on a train.

(3) Sizes of handover signaling messages: The size is the amount of handover signaling messages, proportional to the bandwidth consumption.

(4) Packet loss: The total numbers of lost packets is computed during the handover procedure. The high handover failure probability results in high packet loss and low user satisfaction.

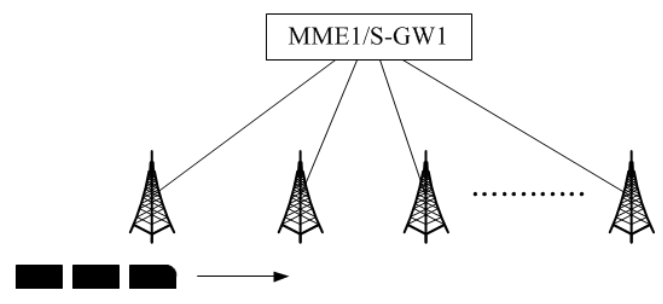

(a) Scenario 1

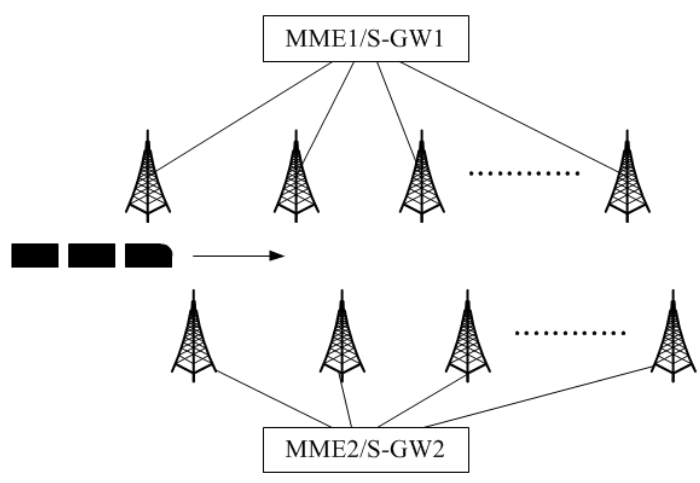

(b) Scenario 2

Fig. 6. Two simulation scenarios: (a) a simple environment (b) a complex environment 
TABLE I

SIMULATION PARAMETERS

\begin{tabular}{|c|c|}
\hline Parameters & Values \\
\hline Transmission range of eNB & $5 \mathrm{~km}$ \\
\hline Number of active users & 1001000 \\
\hline Number of eNBs & 20,40 \\
\hline Velocity of train & $180-360(\mathrm{~km} / \mathrm{h})$ \\
\hline Route advertisement interval & $500 \mathrm{~ms}$ \\
\hline Carrier frequency & $2 \mathrm{GHz}$ \\
\hline Number of OFDM symbols /slot & 7 \\
\hline Multipath & Rayleigh fading channel \\
\hline Length of train & $0.3 \mathrm{~km}$ \\
\hline Transmission power & $85 \mathrm{dBm}(316 \mathrm{~kW})$ \\
\hline Shadow fading deviation & $6 \mathrm{dBm}(0.003981 \mathrm{~W})$ \\
\hline Number of resource blocks & 25 \\
\hline Overlap & $0.4 \mathrm{~km}$ \\
\hline Bandwidth of subcarrier & $15 \mathrm{kHz}$ \\
\hline Packet generation rate per each active user & $10 \mathrm{packets} / \mathrm{s}$ \\
\hline Packet size & $500 \mathrm{bytes}$ \\
\hline Auto-configuration time of IP address & $25 \mathrm{~ms}$ \\
\hline Simulation time & $600 \mathrm{~s}$ \\
\hline
\end{tabular}

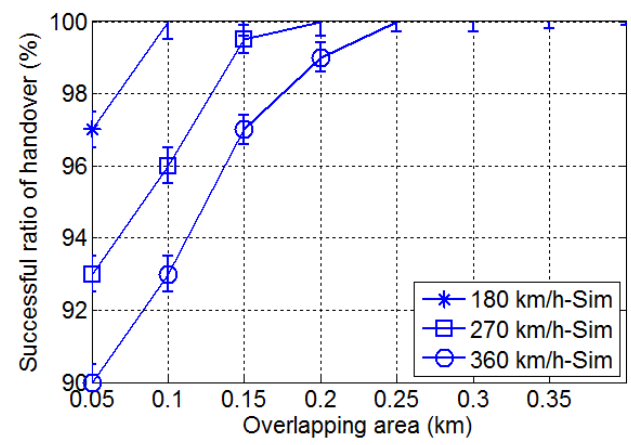

Fig. 7. The successful ratio of handover in different overlapping area

\section{B. Discussion of Overlapping Area}

First, we discuss the overlapping area between two neighbor eNBs, because the overlapping area has a great effect on the successful ratio of the handover procedure. If the handover procedure fails, the throughput of the system will decrease seriously. Figure 7 shows the successful ratio of handover in different overlapping area. We can observe that the smaller overlapping area between two neighbor eNBs could suffer from the handover failure. Moreover, the train has lower successful ratio of handover when it is moving at high speed environment. In other words, the successful ratio of the handover is almost $100 \%$ when the overlapping area is long enough.

\section{Handover Latency}

The handover latency is an important indicator of whether the designed handover procedure is effective. The LTEindividual method only uses a single wireless interface to connect to eNB. Therefore, the average handover latency of the LTE-individual (i.e., $H L_{i d v}$ ) can be formulated as follows:

$$
\begin{array}{r}
H L_{i d v}=\left(1-P_{s_{-} i d v}\right) \times( \\
\left.T_{R e-e n t r y}+T_{R e-c o n n e c t}\right) \\
+P_{s_{-} i d v} \times\left(T_{H O \_} \_i d v\right),
\end{array}
$$

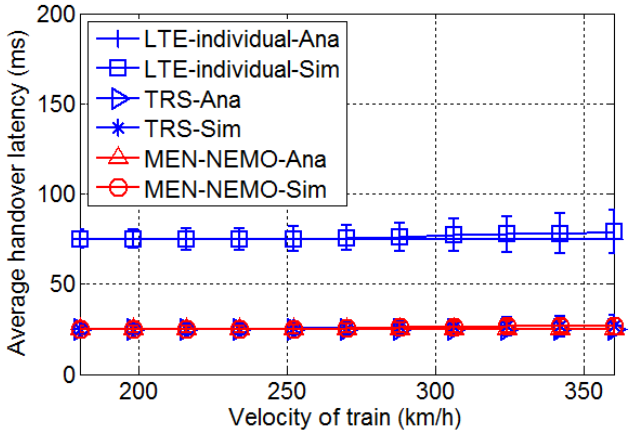

(a)

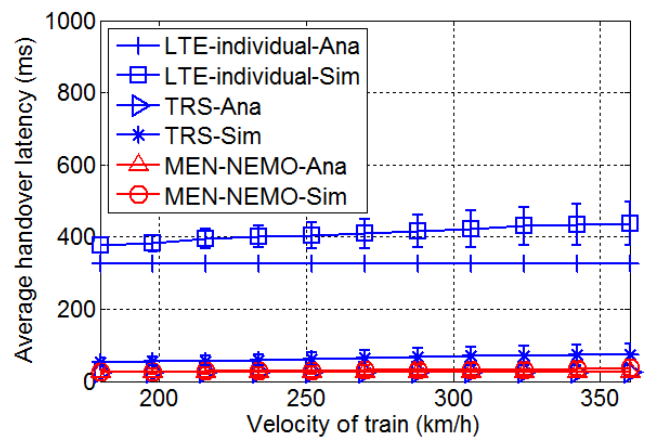

(b)

Fig. 8. The handover latency in: (a) Scenario 1 (b) Scenario 2

where $P_{s_{-} i d v}$ denotes the probability of successful handover for each user under the LTE-individual method. $T_{H O \_} i d v$ is the handover time that the LTE-individual method, requires for the cell scan, negotiation, registration, link switches and auto-configuration of the IP address. Note that the autoconfiguration time of an IP address is zero when the handover is performed in the same IP domain. $T_{R e-e n t r y}$ is the time that the UE requires for the re-entry, and $T_{R e-\text { connect }}$ is the time taken to re-establish an UEs connection if the call was dropped because of a long handover latency. Both the TRS scheme and the MEN-NEMO scheme use multiple antennas scheme to improve the handover procedure. Therefore, the average handover latency of the TRS (i.e., $H L_{T R S}$ ) and that of the MEN-NEMO scheme (i.e., $H L_{\text {Proposed }}$ ) are formulated as follows:

$$
H L_{T R S}=H L_{\text {Proposed }}
$$

$=P_{s} \times\left(T_{H O \_s}\right)+\left(1-P_{s}\right) \times\left(T_{R e-e n t r y}+T_{R e-\text { connect }}\right)$,

where $P_{s}$ denotes the probability of successful handover under the TRS and the MEN-NEMO schemes. $T_{H O \_s}$ is the handover time under both schemes. It includes the time required for link switches and auto-configuration of the IP address. The values of $P_{s_{-} i d v}$ and $P_{s}$ are derived by the scheme in [4] (i.e., $P_{s_{-} i d v}$ is 0.97 and $P_{s}$ is 0.999 when the trains speed is $360 \mathrm{~km} / \mathrm{h}$ ). Moreover, $T_{H O_{-} s}$ is smaller than $T_{H O} i d v$. Figure 8(a) shows the handover latency of three schemes in Scenario 1. The handover delays of the entire schemes are low because the UE/enhanced HeNB does not need to change its IP address. It is noteworthy that the TRS 
and MEN-NEMO schemes yield the best results because they use multiple antennas to reduce the number of disruptions and achieve seamless handover. Figure 8(b) shows the handover latency of three schemes in Scenario 2. The MEN-NEMO scheme outperforms the LTE-individual and the TRS methods because its handover decision policy reduces the number of unnecessary handover operations. The handover latency of the LTE-individual method increases because the UE only uses a single antenna to perform the handover procedure, which includes changing to a new IP address and results in a longer handover delay.

\section{User Satisfaction}

We define a satisfaction score to evaluate the satisfaction levels of UEs on a train. For example, assume there are three MNNs and, in an LTE-individual handover event, two of the MNNs perform handover successfully and one does not. In this case, the satisfaction score of the handover event is $2 r-p$ (i.e., $r$ is the reward and $p$ is the punishment). On the other hand, under the MEN-NEMO scheme, all the handovers performed by the MNNs are either successful or unsuccessful. Given that the number of active users is $\mathrm{n}$, the average satisfaction score of a user (i.e., an MNN) that employs the LTE-individual method can be calculated by binomial formula as follows:

$$
\frac{1}{n} \sum_{k=0}^{n} C_{k}^{n} P_{s_{-} i d v}^{k}\left(1-P_{s_{-} i d v}\right)^{n-k}[k \times r-(n-k) \times p],(3)
$$

where $k$ is the number of successful handovers for active users and is the binomial coefficient. The average satisfaction scores of users that exploit the TRS and the MEN-NEMO schemes are

$$
\frac{1}{n}\left[P_{s} \times n \times r-\left(1-P_{s}\right) \times n \times p\right] .
$$

The average satisfaction scores under different ratios of reward $r$ and punishment $p$ are shown in Figs. 9(a) and 10(a). We assume that $r$ equals 1 and $p$ is set larger than $r$. As the enhanced HeNB can perform handover via the tail antenna if the handover fails via the head antenna, $P_{s}$ is larger than $P_{s \_i d v}$. As a result, the level of user satisfaction is higher under the proposed scheme. An increase in the $p$ score means the user is dissatisfied because of a handover failure. In this situation, the MEN-NEMO scheme maintains a high satisfaction score, but the LTE-individual schemes score decreases rapidly. Figures 9(b) and 10(b) show the average satisfaction scores under different numbers of active users. The MENNEMO scheme still achieves the highest satisfaction score. Moreover, the gap between the LTE-individual-Ana and the LTE-individual-Sim becomes larger when the number of active users increases. Because active users generate large amounts of signaling overhead simultaneously, the eNB becomes a bottleneck and drops a lot of calls.

\section{E. The Size of Handover Signaling Messages}

Table II shows the message sizes of a handover event, assuming there are 100 active UEs on the train. The calculation of the message sizes follows the method in [4], [14], [15] and [16]. In the LTE-individual scheme, the 100 active UEs connect to the eNB on the ground directly; thus, they have

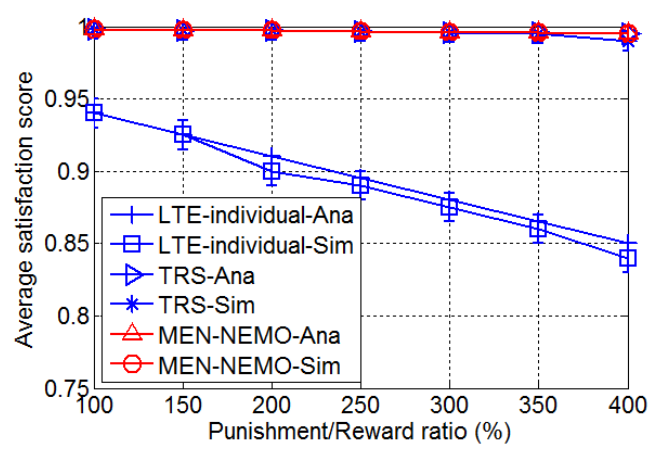

(a)

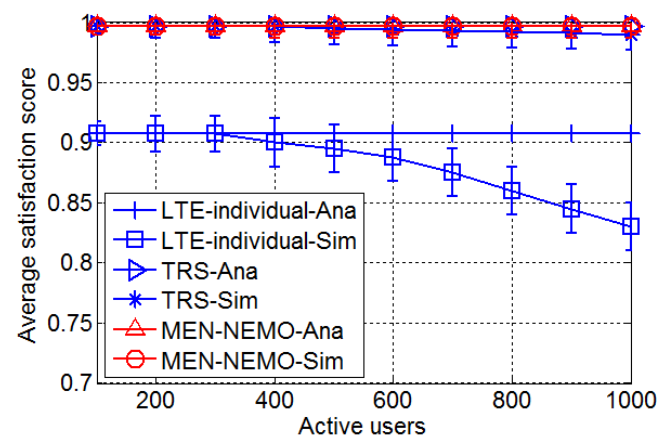

(b)

Fig. 9. The average satisfaction scores in Scenario 1 (a) $(\mathrm{n}=500)(\mathrm{b})($ Punishment/Reward ratio=200\%)

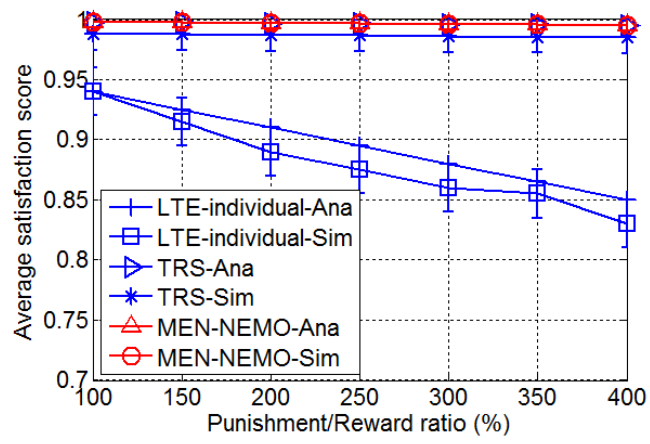

(a)

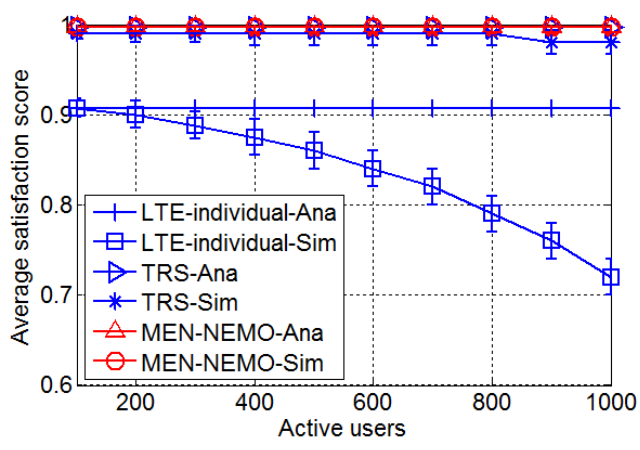

(b)

Fig. 10. The average satisfaction scores in Scenario 2 (a) $(\mathrm{n}=500)$ (b) $($ Punishment/Reward ratio $=200 \%)$ 
This article has been accepted for publication in a future issue of this journal, but has not been fully edited. Content may change prior to final publication. Citation information: DOI 10.1109/TWC.2014.2364179, IEEE Transactions on Wireless Communications

IEEE TRANSACTIONS ON WIRELESS COMMUNICATIONS, VOL. XX, NO. X, 2014

TABLE II

THE SIZES OF HANDOVER SIGNALING MESSAGES

\begin{tabular}{|c|c|c|c|}
\hline & \multicolumn{3}{|c|}{ Signaling Size (Bytes) } \\
\hline Methods & LTE-individual & TRS & MEN-NEMO \\
\hline Measurement Report & 4400 & 44 & 44 \\
\hline Handover Request & 24100 & 15982 & 241 \\
\hline Handover Request Ack & 7000 & 2713 & 70 \\
\hline RRC Conn. Reconf. & 3600 & 36 & 36 \\
\hline RRC Conn. Reconf. Complete & 900 & 9 & 9 \\
\hline SN Status Transfer & 7300 & - & - \\
\hline Path Switch Request & 13600 & 5977 & 136 \\
\hline Path Switch Request Ack & 11700 & 8730 & 117 \\
\hline Modify Bearer Request & 16500 & 4521 & 165 \\
\hline Modify Bearer Response & 8000 & 4337 & 80 \\
\hline UE Context Release & 3000 & 30 & 30 \\
\hline Bi-casting Request & - & 2977 & - \\
\hline Bi-casting Request Ack & - & 3000 & - \\
\hline Total & 75600 & 39498 & 683 \\
\hline
\end{tabular}

to perform handover by themselves. The TRS scheme only requires some messages to contain the TRSs information, such as the Measurement Report. However, some messages (e.g., Handover Requests) still include information about all the active UEs resulting in the message size cannot be compressed. In the proposed scheme, because the enhanced HeNB encapsulates all the UEs traffic, all messages only need to include the information about the enhanced HeNB; therefore, the total message size is further reduced.

Figures 11 and 12 illustrate, respectively, the advantages of using the femtocell-based configuration and the MR. The main difference between Fig. 11 and Fig. 12 is the signaling overhead incurred by changing the IP address. Note that the LTE-individual scheme exchanges a large number of signaling messages to configure the new IP address in Scenario 2. In the MEN-NEMO scheme, the enhanced HeNB uses the MR function to reduce the signaling overhead. Therefore, the total message size remains low when the number of active users increases.

\section{F. Packet Loss}

As shown in Figure 13 (a) and (b), MEN-NEMO scheme outperforms the other schemes in terms of packet losses. The LTE-individual scheme loses lots of packets because 1) the UE only uses one interface to perform the handover procedure resulting in higher handover failure probability and 2) the higher handover latency causes more lost packets. In Scenario 2 , the lost packets of the LTE-individual scheme increases obviously since the train may change its IP address and increase handover latency. However, TRS and MEN-NEMO still have few lost packets. This results show the advantage of multiple antennas scheme on the train.

\section{CONCLUSIONS AND FUTURE WORK}

The designs of network mobility scheme for high-speed rail systems are differentiated by the train-to-ground wireless access technologies, the network architecture and infrastructure on the train, the MR design, the impact on end-devices, and the handover protocol. This paper proposed a LTE femtocellbased network mobility scheme which only requires minor

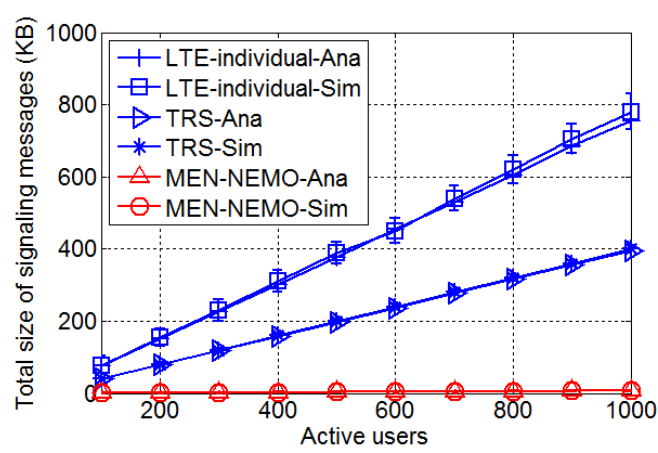

Fig. 11. The total size of signaling messages with different number of active users in Scenario 1

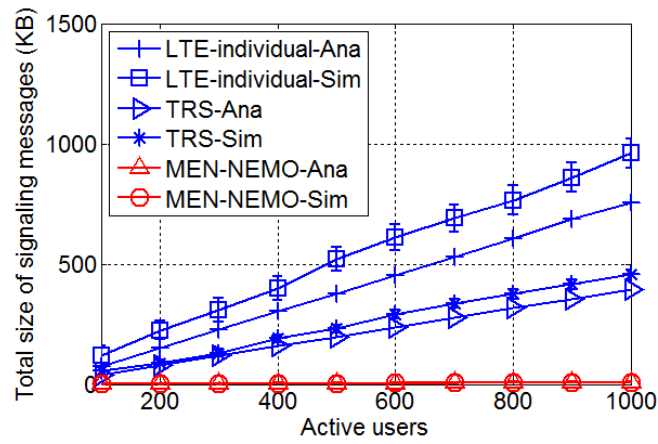

Fig. 12. The total size of signaling messages with different number of active users in Scenario 2

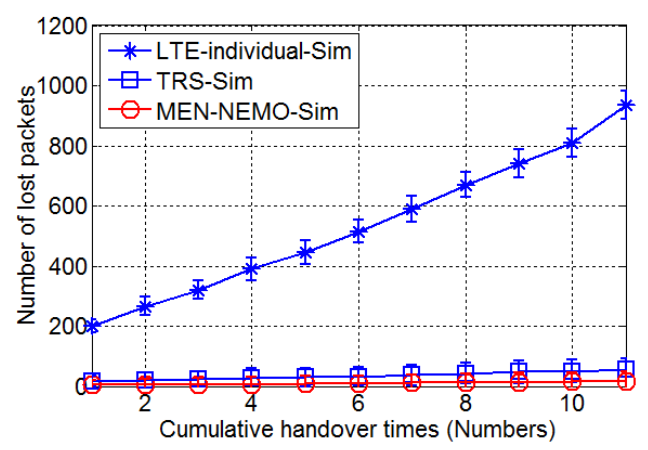

(a)

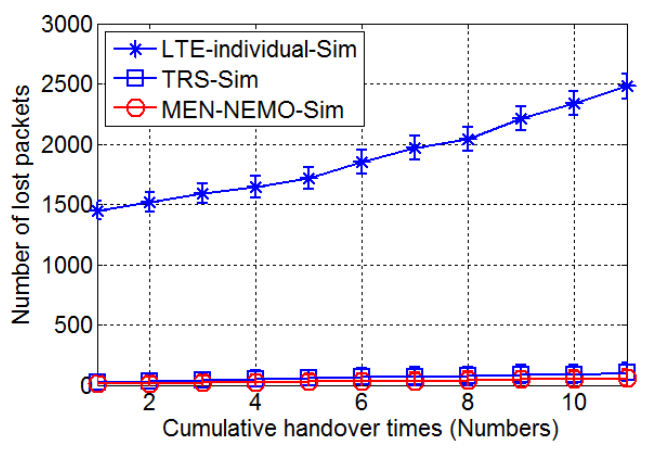

(b)

Fig. 13. The performance of the packet loss 
modification of the eNB, so it is easy to deploy. Two antennas are mounted on the train, one on the head carriage and another on the tail carriage. They alternate the execution of handovers and transmission of the mobile networks traffic. Therefore, the proposed handover protocol provides uninterrupted service for the MNNs, which are important for communications on high-speed trains. The proposed scheme was evaluated using simulation and analysis, and the performance analysis shows that the MEN-NEMO scheme outperforms the other schemes. Resource management is also an important issue because wireless spectrum is a finite resource. Therefore, in a future work, we will consider resource management for different types of traffic (e.g., voice, audio and video traffic) to maximize the system utilization in a high-speed train environment.

\section{REFERENCES}

[1] D.T. Fokum and V.S. Frost, A Survey on Methods for Broadband Internet Access on Trains, IEEE Communications Surveys \& Tutorials, vol. 12, no. 2, pp. 171-185, Apr. 2010.

[2] T. Ernst and H.-Y. Lach, Network Mobility Support Terminology, IETF RFC 4885, Jul. 2007.

[3] C.-W. Lee, T.-Y. Lee, M. C. Chen and Y. S. Sun, An Integrated Network Mobility Management and Call Admission Control Scheme for Internet Access on High-speed Trains, Technical Report TR-IIS-11-004, Institute of Information Science, Academia Sinica, Jul. 2011.

[4] L. Tian, J. Li, Y. Huang, J. Shi and J. Zhou, Seamless Dual-Link Handover Scheme in Broadband Wireless Communication Systems for High-Speed Rail, IEEE Journal on Selected Areas in Communications (JSAC), vol. 30, no. 4, pp. 708-718, May 2012.

[5] S. Herborn, L. Haslett, R. Boreli and A. Seneviratne, HarMoNy - HIP Mobile Networks, Proc. IEEE Vehicular Technology Conference (VTC 2006-Spring), pp. 871-875, May 2006.

[6] C.-M. Huang, C.-H. Lee and J.-R. Zheng, A Novel SIP-Based Route Optimization for Network Mobility, IEEE Journal on Selected Areas in Communications, vol. 24, no. 9, pp. 1682-1691, Sep. 2006.

[7] Y.-C. Tseng, J.-J. Chen and Y.-L. Cheng, Design and Implementation of a SIP-Based Mobile and Vehicular Wireless Network With Push Mechanism, IEEE Transactions on Vehicular Technology, vol. 56, no. 6, pp. 3408-3420, Nov. 2007.

[8] V. Devarapalli, R. Wakikawa, A. Petrescu and P. Thubert, Network Mobility (NEMO) Basic Support Protocol, IETF RFC 3963, Jan. 2005.

[9] P. Rodriguez, R. Chakravorty, J. Chesterfield, I. Pratt and S. Banerjee, MAR: a commuter router infrastructure for the mobile Internet, Proc. International Conference On Mobile Systems, Applications And Services (MobiSys), pp. 217-230, Jun. 2004.

[10] O.B. Karimi, J. Liu and C. Wang, Seamless Wireless Connectivity for Multimedia Services in High Speed Trains, IEEE Journal on Selected Areas in Communications(JSAC), vol. 30, no. 4, pp. 729-739, May 2012.

[11] 3GPP TS 36.300: Overall description; Stage 2, V11.2.0, Jun. 2012.

[12] 3GPP TS 23.401: General Packet Radio Service (GPRS) enhancements for Evolved Universal Terrestrial Radio Access Network (E-UTRAN) access, V11.3.0, Sep. 2012.

[13] 3GPP TS 32.593: Home enhanced Node B (HeNB) Operations, Administration, Maintenance and Provisioning (OAM\&P); Procedure flows for Type 1 interface HeNB to HeNB Management System (HeMS), V11.0.0, Sep. 2011

[14] 3GPP TS 36.331: Radio Resource Control (RRC); Protocol specification, V11.1.0, Sep. 2012.

[15] 3GPP TS 36.413: S1 Application Protocol (S1AP), V11.1.0, Sep. 2012.

[16] 3GPP TS 36.423: X2 application protocol (X2AP), V11.2.0, Sep. 2012.

[17] The Network Simulator 3 (NS3), Available: http://www.nsnam.org/.

[18] Ming-Chin Chuang and Jeng-Farn Lee, A Lightweight Mutual Authentication Mechanism for Network Mobility in IEEE 802.16e Wireless Networks, Computer Networks, Vol.55, Issue 16, pp. 3796-3809, Nov. 2011.

[19] Ming-Chin Chuang and Jeng-Farn Lee, DRO: Domain-Based Route Optimization Scheme for Nested Mobile Networks, EURASIP Journal on Wireless Communications and Networking, pp. 1-19, Aug. 2011.

[20] 3GPP, TR 36.814 (V9.0.0), Mar. 2010.

[21] D. Huang, P. Fan, and K. B. Letaief, An optimal antenna assignment strategy for information raining, IEEE Transactions on Wireless Communications, vol. 7, no. 4, pp. 1134 - 1139, Apr. 2008.
[22] C. Sue, S. Sorour, Y Yuk, and S. Valaee, Network Coded Information Raining over High-Speed Rail through IEEE 802.16j,' IEEE Personal, Indoor, Mobile, Radio Communication Conference (PIMRC), pp. 11381142, Sep. 2009.

[23] D. Ho and S. Valaee, Information Raining for Link-Layer Design of Mobile Hotspots, IEEE Trans. on Mobile Computing, vol. 4, no. 3, pp. 271-284, May 2005.

[24] 3GPP TR 36.871, Evolved Universal Terrestrial Radio Access (EUTRA); Downlink Multiple Input Multiple Output (MIMO) enhancement for LTE-Advanced, version 11.0.0, Sep. 2012.

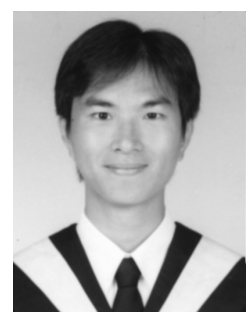

Cheng-Wei Lee received the B.S., M.B.A. and $\mathrm{Ph} . \mathrm{D}$. degree from the Department of Information Management, National Taiwan University in 2002, 2004 and 2011 respectively. He was with Research Center for Information Technology Innovation, Academia Sinica, Taiwan as a Postdoctoral Fellow from 2011 to 2012. His current research interests include mobile Internet, vehicular networks, mobility management, cellular communication systems, and wireless mesh networks.

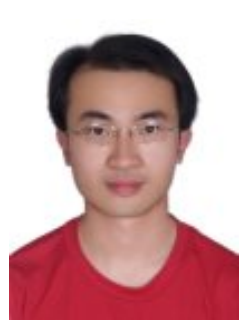

Ming-Chin Chuang received the Ph.D. degree in the Department of Computer Science and Information Engineering from National Chung Cheng University, Taiwan, in 2012. He is a Postdoctoral Fellow in the Institute of Information Science, Academia Sinica, Taiwan. His research interests include mobility management, network security, cloud computing, and VANET.

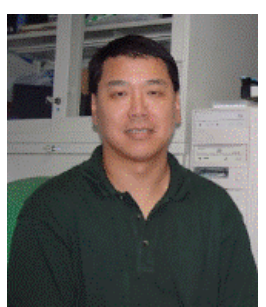

Meng Chang Chen received the Ph.D. degree in Computer Science from the University of California, Los Angeles, in 1989. He was with AT\&T Bell Labs from 1989 to 1992, New Jersey, USA and now he is a Research Fellow of Institute of Information Science, Academia Sinica, Taiwan. His current research interests include wireless network, QoS networking, information retrieval, data and knowledge engineering.

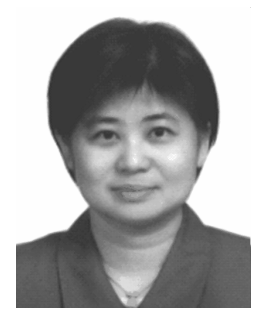

Yeali S. Sun received her BS degree in Computer Science from National Taiwan University, and the MS and Ph.D. degrees in Computer Science from the University of California, Los Angeles (UCLA) in 1984 and 1988, respectively. From 1988 to 1993, she was with Bell Communications Research Inc. (Bellcore; now Telcordia), where she was involved in the area of planning and architecture design of information networking, broadband networks, and network and system management. In August 1993, she jointed National Taiwan University and is currently a professor of the Department of Information Management. She is the director of the Computer Center of National Taiwan University. In 19962002, she served in the TANet Technical Committee, Steering Committee of the National Broadband Experimental Network (NBEN) and Internet2, and IP Committee of TWNIC. Her research interests are in the area of mobile Internet, quality of service (QoS), content classication, wireless mesh networks, multimedia content delivery, Internet pricing and network management, and performance modeling and evaluation. 